\title{
Exploring Patient Safety Culture in a Kuwaiti Secondary Care Setting A qualitative study
}

"Hamad Alqattan, ${ }^{1}$ Jennifer Cleland, ${ }^{2}$ Zoe Morrison, ${ }^{3}$ Isobel M. Cameron ${ }^{2}$

$$
\text { استكشاف ثقافة سلامة المريض في مرافق الرعاية الثانوية الكويتية }
$$

حمد القطان، جينيفر كليلاند، زوي موريسون، إيزبيل كاميرون

ABSTRAC T: Objectives: Qualitative studies can improve understanding of patient safety culture (PSC), which has been relatively neglected by researchers in the Gulf Cooperation Council context. This study employed a qualitative approach to explore healthcare staff and patients' perceptions of PSC and how it can be improved. Methods: This qualitative study was conducted in a public hospital in Kuwait. Individual face-to-face interviews were used to understand the experience of healthcare staff and patients concerning PSC. After obtaining the required ethical approvals, maximum variation sampling was used. Interviews were recorded and transcribed. The analysis was inductive and thematic. Results: A total of 51 participants were included in this study ( 35 healthcare professionals and 16 patients). Data analysis revealed four overarching themes relevant to the research question: (1) workload; (2) communication; (3) environmental constraints; and (4) incident reporting. These issues were interrelated in practice. Kuwaiti and non-Kuwaiti participants held different views, particularly about the response to errors and expatriate staff members' clinical skills. Conclusion: This study revealed multiple factors related to workload, communication, healthcare environment and incident reporting, which hindered the promotion of positive PSC in the included department. The presence of numerous constraints suggests that multiple interventions which target both individual and organisational levels should be implemented.

Keywords: Health Services Research; Patient Safety; Safety Culture; Kuwait.

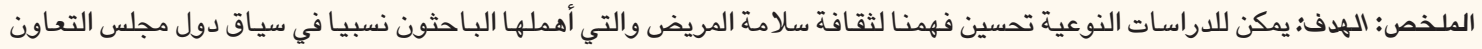

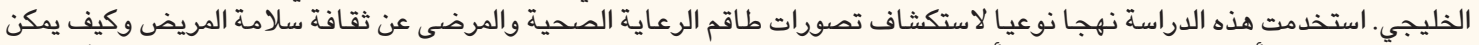

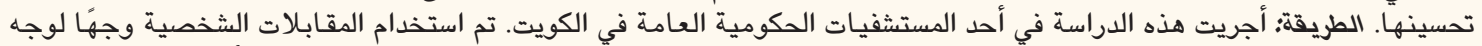

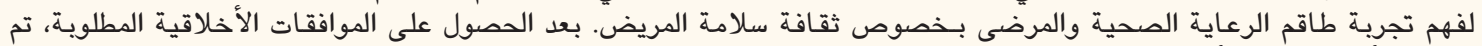

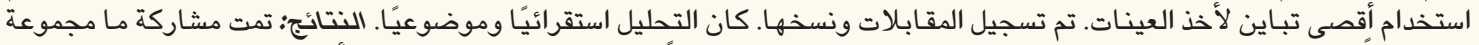

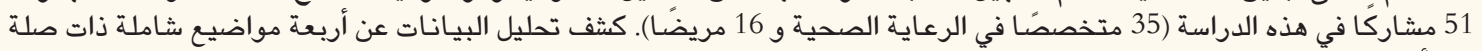

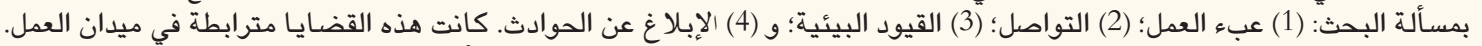

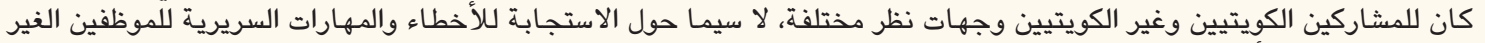

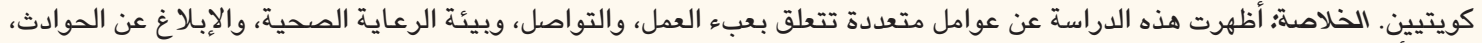

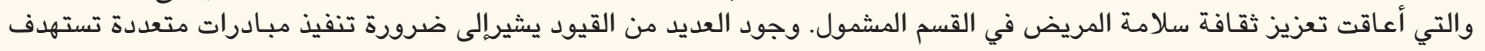
المستويين الفردي والتنظيمي.

الكلمات المفتاحية: بحوث الخدمات الصحية؛ سلامة المريض؛ الثقافة ؛ السلامة؛ الكويت.

\section{Advances In KNOWledge}

This study found that the obstacles that prevent an optimal patient safety culture (PSC) in a hospital setting in Kuwait are associated with workload, communication, environmental constraints and incident reporting.

\section{Application to Patient Care}

The healthcare environment and how the staff behaves and communicates with patients play a crucial role in determining patients' perceptions of the department's PSC.

$\mathrm{M}$ ANy patient SAFEty CUlture (PSC) studies have been carried out in Kuwait and other Gulf Cooperation Council (GCC) countries using the Hospital Survey on Patient
Safety Culture (HOSPSC). ${ }^{1,2}$ Collectively, these studies found that the overall punitive response to errors, shortness of staff and lack of open communication hindered the development of optimal PSC among 
healthcare professionals. ${ }^{2}$ The presence of a culture which promotes patient safety is critical for improving patients' outcomes in healthcare organisations. However, research suggests that PSC differs across countries. ${ }^{3}$ It was also evident from the literature that PSC has been studied in greater depth in some countries than others. For example, a narrative synthesis of 22 qualitative and mixed methods PSC studies revealed that none had been conducted in Arab contexts. ${ }^{4}$ Since this review, only one qualitative study in the Arab/GCC context was published. ${ }^{5}$ This recent study explored doctors' and nurses' PSC perspectives in two public hospitals in Kuwait. The authors concluded that participants had a good understanding of the concept of PSC and that appropriate leadership support, resources and response to errors were required to achieve optimal PSC levels in the studied context. ${ }^{5}$

Surveys describe a phenomenon at a particular point in time. Still, they cannot provide an understanding as to 'why', i.e. gaining an understanding of how healthcare staff and patients experience PSC in the day-to-day world of healthcare delivery. However, such knowledge can help decision-makers identify factors that may hinder safe healthcare services and reveal areas to target for intervention. ${ }^{6}$ While a quantitative study can identify and measure PSC issues, qualitative ones can explore specific factors contributing to these issues and potential ways to address them.

Patient perspectives were seldomly considered in PSC studies. ${ }^{4}$ However, patients are at the centre of the service delivery process and are an ideal source of information on PSC. ${ }^{8}$ Patients also see PSC from a different perspective than healthcare professionals. ${ }^{9}$ This different perspective suggests that obtaining the views, attitudes and experiences of both healthcare professionals and patients may provide a more comprehensive picture of PSC in a hospital setting. Although there have been many PSC studies, this area has been relatively neglected in GCC contexts, particularly through qualitative approaches. This study aimed to identify and explore staff and patient perceptions of factors that hinder optimal PSC implementation and how these factors might be addressed. This study builds on an earlier survey of staff perceptions of PSC. ${ }^{10}$

\section{Methods}

This qualitative study was conducted using face-toface interviews with participants from the medical department of a public hospital in Kuwait between June and August 2018. This department was selected as it provides a wide range of medical services, has employees of different nationalities, and was working to implement the Kuwaiti National Patient Safety Standards at the time of the study. ${ }^{11}$

Maximum variation purposive sampling was employed to include a wide range of healthcare professionals and patients. Healthcare professionals who had worked in the department for less than a year were excluded to ensure participants had had sufficient experience with and knowledge about the department to engage fully with the research questions. Patients who were admitted to the medical wards or visited the medical outpatients' clinics were also interviewed. Participants were given interviews in either Arabic or English to ensure the most comprehensive dialogue possible based on the participants' preference. ${ }^{12}$ It should be noted that English was not the first language of all participants.

The relevant department heads nominated a facilitator whose role was to explain the study's aim and voluntary nature to their department's staff and patients and coordinate interviews. The first author conducted all interviews. Before starting the interviews, he reiterated the study's purpose, invited questions and obtained written consent. Potential participants were reassured that they had the right to withdraw from the study at any time without prejudice or consequence.

Interview questions were drawn from the PSC literature in the field and informed by an earlier survey conducted in the same department.,10 Openended questions about factors affecting PSC in the department were used to guide the discussions, with follow-up questions from the researcher to obtain a deeper understanding of the participants' views. Participants were also asked to speculate on strategies to improve PSC. Interviews continued until each participant felt they had sufficiently shared their experiences.

Notes and audio recordings were reviewed after each interview so that the content of earlier interviews could expose further questions for later interviews. ${ }^{13,14}$ This process was used to check for data saturation, which was estimated to have occurred after interviews with 25 healthcare professionals and 16 patients; however, 10 further healthcare professionals were interviewed to ensure a full range of views across all professional groups. ${ }^{15}$

The interview recordings were transcribed verbatim. The recordings and transcribed data were stored on a password-protected university computer, while written notes and data were stored in a locked cabinet on university premises. Only the authors/ research team had access to the data. 
The qualitative content analysis approach of Graneheim and Lundman guided the data analysis. ${ }^{16}$ As is typical for qualitative data analysis, the approach was non-linear. The researchers moved back and forth through the analytical stages of specifying and defining the unit of analysis, sorting the unit of analysis into content areas, dividing content areas into meaning units, labelling meaning units with codes, clustering the coded meaning units into subthemes and clustering the subthemes into themes. ${ }^{16}$

The first step was to read the transcripts several times to become familiar with the data. The unit of analysis was the text obtained from the interviews' transcripts. Using Microsoft Word 365 (Microsoft, Corp., Redmond, Washington, USA), the text was sorted into two content areas based on two research questions: (1) "What factors hamper promoting PSC?"; and (2) "What interventions might improve PSC?". Arabic texts within each content area were first translated into English and then entered into NVivo, Version 11 (QSR International, Doncaster, Australia) for data management and to facilitate data coding and analysis. Each content area was then divided into meaning units.

To standardise coding meaning units across the entire text, the researchers carried out the process in two phases. In the first phase (i.e. preliminary coding), they analysed three interview transcripts to develop an initial coding framework. In the second phase, they applied these finalised codes across the entire text. The whole context was considered while applying the codes to the meaning units. After coding, the researchers searched the meaning units for similarities and differences and clustered them into subthemes. The codes were discussed and reviewed at regular intervals among the researchers to identify and develop themes as well as minimise individual researcher influence during analysis. Coding disagreements were resolved through team discussions. Coding and interpretation occurred iteratively and inductively, focusing throughout on the research aim.

In this study, the credibility and dependability of the findings were improved by obtaining the views of a wide range of participants to help provide more substantial evidence and a better description of the results. ${ }^{17}$ Audio-recordings and transcribing interviews verbatim improved accuracy and precision in presenting participants' views. The transferability of this study was addressed by providing detailed descriptions of the data collection and analysis processes. ${ }^{18}$ In addition, the study's findings were frequently discussed among the research team, which ensured to draw conclusions only once consensus was achieved to guarantee the findings' confirmability. ${ }^{17}$

Written ethical approval was obtained from the Ministry of Health of Kuwait, the hospital director and the departments' heads (2017/582). Informed consent was also obtained from all participants.

\section{Results}

In total, 35 healthcare professionals and 16 patients were interviewed for this study. Most healthcare professionals were non-Kuwaitis $(\mathrm{n}=21)$ and male $(\mathrm{n}=19)$. Conversely, most patients were Kuwaiti ( $\mathrm{n}=14)$ and female $(\mathrm{n}=13)$. Most participants preferred to be interviewed in Arabic ( $\mathrm{n}=34$ ) [Table 1]. The average interview duration was 30 minutes. Data analysis revealed four predominant themes relevant to the research question: (1) workload; (2) communication; (3) environmental constraints; and (4) incident reporting [Table 2].

All staff participants raised the issue of heavy workloads hindering the provision of safe medical services in the department. The data indicated the presence of four major factors contributing to the

Table 1: Characteristics of included healthcare professionals and patients at a public hospital in Kuwait $(\mathrm{N}=51)$

\begin{tabular}{|c|c|c|c|c|c|c|c|}
\hline \multirow[t]{2}{*}{ Participant jobs } & \multirow[t]{2}{*}{ Number } & \multicolumn{2}{|c|}{ Nationality } & \multicolumn{2}{|c|}{ Gender } & \multicolumn{2}{|c|}{$\begin{array}{c}\text { Language of } \\
\text { interviews }\end{array}$} \\
\hline & & Kuwaiti & Non-Kuwaiti & Male & Female & Arabic & English \\
\hline Medical doctor & 6 & 2 & 4 & 6 & 0 & 5 & 1 \\
\hline Registered nurse & 13 & 2 & 11 & 8 & 5 & 3 & 10 \\
\hline Pharmacist & 5 & 3 & 2 & 2 & 3 & 5 & 0 \\
\hline Nuclear medicine doctor & 3 & 3 & 0 & 0 & 3 & 2 & 1 \\
\hline Laboratory technician & 4 & 3 & 1 & 1 & 3 & 2 & 2 \\
\hline
\end{tabular}


Table 2: Summary of participants' concerns and suggested solutions from a public hospital in Kuwait

Themes

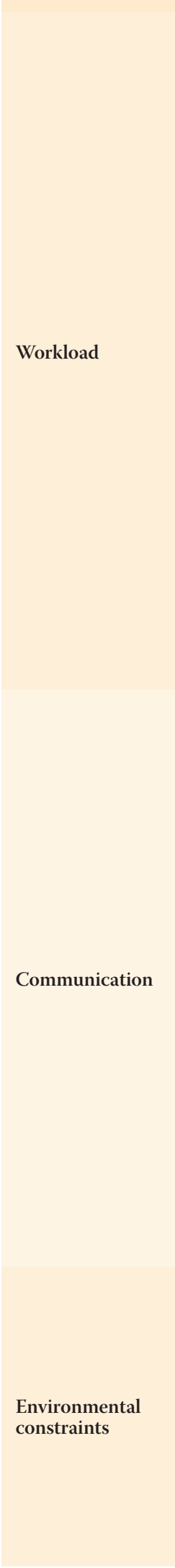

\section{Concerns}

Number of staff $(\mathrm{S})$

\section{Professional} competence $(\mathrm{S}+\mathrm{P})$

Impact of visitors (S)

\section{Appropriateness} of admissions (S)

Interdepartmental cooperation (S)

Appropriateness of documentation (S)

Reliance on junior staff (S)

Healthcare professionals' behaviour $(\mathrm{P})$

Hygiene $(\mathrm{S}+\mathrm{P})$

Appropriateness of facilities (S)
Suggested solution(s)

- Increase numbers of staff

- Provide paid overtime

- Establish another hospital to serve as a catchment area

- Ongoing staff orientation on patient safety

- Assess pre-employment competency in patient

safety for medical department staff

- Offer competitive wages and incentives

Implement strict ward visiting hours for patient families

Establish intermediate care units/wards

Develop interdepartmental policies

Offer ongoing staff orientation on appropriate documentation

Assign only senior/ experienced staff for consultations with other staff

Offer ongoing staff training on communication skills

Clean and maintain facilities regularly/frequently

Expand the department's facilities

\section{Supporting quotes}

- "Our number is less than what is required for this health region. Indeed we have a shortage of staff" (MD2)

- "I told you we are running here. So better they can supply some staff" (RN5).

- "When the nurse came to me, she did not know where to insert the needle. I have bruises on my both arms" (Patient 11)

- "I will try to improve education for the whole staff, involving them in more regular courses or lectures in hospitals. Make sure every staff is involved in orientations" (LAB2).

- "The employment board should be reformed as it is responsible for contracting with expat staff" (MD4).

- "This is madness, why visitors are here for 24 hours. I cannot provide good care in such an atmosphere" (RN5).

- "We can give visiting time between 11 to 12 like that relatives will come, doctor, is there" (RN11).

- "Most of the patients are either bedridden or in mechanical ventilation who are unable to help themselves" (RN7)

- "It is ICU patients. Ventilator patients mean it should be ICU. So in ICU, they are taking one nurse to one. So one ventilator patient means one staff will take care of the patient in the whole shift" (RN7).

- "We have regimens for some medications differ from those with doctors. That is why we clash with them" (PH3).

- "I think there should be frequent meetings between the different healthcare professionals to support multidisciplinary teamwork" (PH4).

- "Most of the prescriptions we receive here in the pharmacy lack some important patients' information, like patient weight" (PH1).

- "The doctor's handwriting is not clear. Usually, I cannot read what is written in the file, so I need to chase the doctor the whole day" (RN3).

- "The trainees should not be dealt with as a competent doctor. They are still under training. We know that it is very risky to rely on them. However, on busy days, we are forced to" (MD5).

- "Any consultation to be sent should be seen by the senior registrar at least. Not allowed to send someone a junior or assistant or registrar to come" (MD3).

- "The doctors, I do not see them, and even when they come, I cannot get what they say" (Patient 4).

- "Somebody should keep reminding them about how to deal with patients" (Patient 8).

- "Even the place is not clean enough. In the past, when we go to hospitals, the smell of Dettol was very clear but nowadays it is not" (Patient 2).

- "I think it is the role of supervisors to come and see the condition of the wards themselves" (PH5).

- "The toilet smells very bad. The washing sink is leaking. I told them about it two days ago, but nobody cares" (Patient 14)

- "We said to the nurses that these medications should be stored in a room temperature of between $20^{\circ} \mathrm{C}$ and $25^{\circ} \mathrm{C}$. However, they always say that air conditioning is not working well, and nobody fixes it yet" (PH4).

$S=$ indicated by staff participants; $M D=$ medical doctor $R N=$ registered nurse; $S+P=$ indicated by staff and patient participants; $L A B=$ laboratory technician; $I C U=$ intensive care unit; $P H=$ pharmacist; $P=$ indicated by patient participants; $P T=$ physiotherapist; NM = nuclear medicine doctors 
Table 2 (cont'd): Summary of participants' concerns and suggested solutions from a public hospital in Kuwait

\begin{tabular}{|c|c|c|c|}
\hline Themes & Concerns & Suggested solution(s) & Supporting quotes \\
\hline \multirow[t]{2}{*}{$\begin{array}{l}\text { Environmental } \\
\text { constraints }\end{array}$} & $\begin{array}{l}\text { Availability of } \\
\text { equipment and } \\
\text { supplies }(\mathrm{S}+\mathrm{P})\end{array}$ & $\begin{array}{l}\text { Make equipment and } \\
\text { supplies consistently } \\
\text { available }\end{array}$ & $\begin{array}{l}\text { - "We need some important equipment which is not } \\
\text { available in medical wards like some special beds and } \\
\text { lifters. So, we are always forced to transfer patients } \\
\text { from medical building to our department in the old } \\
\text { building" (PT1). } \\
\text { - "Once, we waited for around three months to get ink, } \\
\text { and now we are almost out of A4 papers" (MD5). }\end{array}$ \\
\hline & $\begin{array}{l}\text { Privacy and } \\
\text { security }(\mathrm{P})\end{array}$ & $\begin{array}{l}\text { Increase the number of } \\
\text { private rooms }\end{array}$ & $\begin{array}{l}\text { - "I am not safe here. The next patient always cough. } \\
\text { My health is deteriorating here" (Patient 2). } \\
\text { - "I want a private room. I do not want to bother } \\
\text { anybody and do not want anybody to bother me" } \\
\text { (Patient 16). }\end{array}$ \\
\hline \multirow[t]{4}{*}{$\begin{array}{l}\text { Incident } \\
\text { reporting }\end{array}$} & $\begin{array}{l}\text { Response to } \\
\text { errors }(\mathrm{S}+\mathrm{P})\end{array}$ & $\begin{array}{l}\text { Promote/reward staff } \\
\text { who participate in quality } \\
\text { improvement and patient } \\
\text { safety initiatives }\end{array}$ & $\begin{array}{l}\text { - "Completing an incident report may ruin my } \\
\text { relationship with my friends and colleagues" (LAB2). } \\
\text { - "I am threatened by the aggressive attitude of the } \\
\text { patients and those responsible for them. I always } \\
\text { feel that I am guilty, and they are so motivated to } \\
\text { complain" (RN5). } \\
\text { - "We never punish anyone in here - when our staff } \\
\text { make mistakes, we talk with them in a friendly way" } \\
\text { (NM3). } \\
\text { - "Yesterday, nurses forgot to give me the medication. } \\
\text { She should be fired" (Patient 3). } \\
\text { - "As a leader, I should be the first one to attend } \\
\text { patient safety lectures. I will not ask the staff to attend } \\
\text { without my presence. I should be there to support the } \\
\text { staff" (RN9). }\end{array}$ \\
\hline & \multirow{2}{*}{$\begin{array}{l}\text { Feedback and } \\
\text { follow-up } \\
\text { after reporting } \\
\text { incidents }(\mathrm{S})\end{array}$} & $\begin{array}{l}\text { - Simplify the incident report } \\
\text { form }\end{array}$ & \multirow{2}{*}{$\begin{array}{l}\text { - "We do not know what the reaction is because we } \\
\text { completed many forms since one year, but we did not } \\
\text { see any reaction" (RN7). } \\
\text { - "We never heard about what happened afterwards, } \\
\text { so I wrote the paper, the paper was written, it was } \\
\text { forwarded, full stop" (RN1). }\end{array}$} \\
\hline & & $\begin{array}{l}\text { - Establish a system of } \\
\text { feedback after reporting an } \\
\text { incident }\end{array}$ & \\
\hline & $\begin{array}{l}\text { Appropriateness } \\
\text { of documentation } \\
\text { (S) }\end{array}$ & $\begin{array}{l}\text { Offer ongoing staff } \\
\text { orientation on appropriate } \\
\text { documentation }\end{array}$ & $\begin{array}{l}\text { - "Most of the prescriptions we receive here in the } \\
\text { pharmacy lack some important patients' information, } \\
\text { like patient weight" (PH1). } \\
\text { - "The doctor's handwriting is not clear. Usually, I } \\
\text { cannot read what is written in the file, so I need to } \\
\text { chase the doctor the whole day" (RN3). }\end{array}$ \\
\hline
\end{tabular}

$S=$ indicated by staff participants; $M D=$ medical doctor; $R N=$ registered nurse; $S+P=$ indicated by staff and patient participants; $L A B=$ laboratory technician; $I C U=$ intensive care unit $P H=$ pharmacist $P=$ indicated by patient participants; $P T=$ physiotherapist; NM = nuclear medicine doctors.

heavy workload. Three of these were raised by staff participants, not patients: (1) number of staff; (2) the impact of visitors; and (3) the appropriateness of admissions. Professional competence was raised as a factor contributing to the heavy workload by both staff and patients.

The staff believed it was difficult to provide medical services at the required standard with the current staff-to-patient ratio. This challenging ratio had been exacerbated by many healthcare personnel, particularly the nursing staff, either resigning or being transferred to other departments without replacement. The combination of high patient numbers and staff shortages was considered relevant to patient safety since the staff discussed how they were forced to take short-cuts to finish their tasks on time. To address this challenge, the staff suggested that the hospital administration prioritise increasing staff numbers. Paid overtime was additionally seen as a possible solution. Some even suggested the need for another hospital to assist in providing healthcare services in the region and alleviating heavy workloads.

Both Kuwaiti patients and staff expressed the opinion that expatriate staff lacked basic clinical skills. This perceived lack of skills and underperformance meant Kuwaiti staff members thought it was easier to do tasks themselves rather than engaging with an expatriate staff member. A related issue was high staff turnover, particularly the nursing staff, as new staff would lack familiarity with work procedures. This lack of familiarity could mean additional burden for staff members who had been in the department longer to do most tasks themselves and spend most of their time training new staff on work procedures. Training and orientation were seen by the staff as a potential solution to this problem, as well as offering competitive wages and incentives to attract more competent staff. Performing a pre-employment competency assessment with the medical staff was seen by many patients as a potential solution. 
The nursing staff and those working in the wards stressed the importance of implementing strict visiting hours for patients' families. They claimed that the presence of patients' relatives and family members after visitation hours was detrimental to good quality care, causing loss of concentration and delay in services. The nature of patient presentations was cited as contributing to heavy staff workloads. Staff participants believed that many patients needed constant close observation and special care, which added to the nursing staffs' workload. To address this problem, many staff participants suggested providing an intermediate critical care unit for suitable admissions.

Three interrelated issues were reported as negatively affecting communication across the different professional groups. The first issue was that different professional groups had conflicting policies and procedures, which resulted in frequent disagreements and delays in service provision including laboratory results and medication administration. The second issue raised was the lack of appropriate record-keeping and documentation, which hindered communication between the staff members. The third issue was related to an overreliance on inexperienced junior staff for consultations because of staff shortages. This issue meant that staff members were expected to work above their level of competence. Staff participants reported that developing interdepartmental policies and communication systems, such as regular meetings, ongoing staff orientation on appropriate documentation in the medical records and assigning only senior/experienced staff members for consultations, would improve cooperation between the different specialities and support multidisciplinary healthcare provision.

Several patients commented on staff behaviour, reporting, for example, that staff did not take into consideration patient preferences and that patients were not reasonably involved in healthcare plans. Moreover, patients complained about some doctors' and nurses' rude attitudes. Patients believed that these issues could be addressed by better departmental leadership, intensifying staff orientation and offering ongoing training, particularly in staff-patient communication skills.

Both the staff and patients frequently raised their dissatisfaction with the healthcare environment. Participants mentioned numerous problems related to the healthcare environment including hygiene, facilities, equipment, supplies and privacy. All patients and some staff members thought that the medical wards were not sufficiently clean. There was a shortage of essential equipment including beds and physiotherapy kits and necessary supplies such as hand sanitisers and patient diapers. Furthermore, existing facilities were poorly maintained and much of the equipment was broken. Both the staff and patients reported that these problems could be overcome through regular and frequent cleaning and maintenance and ensuring consistent availability of equipment and supplies.

Space was also an issue for the staff, particularly those working in outpatient clinics. Both the lack of clinical space and the inappropriateness of available space was noted. According to staff members, the lack of such special rooms in the medical facility made the process of taking care of patients overwhelming for both patients and healthcare professionals. They believed that a large expansion, which included all the department's premises, should be initiated to overcome this problem.

Many patients admitted to the medical wards' shared rooms expressed deep upset and distress at disturbances caused by nearby patients and their relatives. They also worried about getting hospitalacquired infections from other patients in the same room due to the environment's poor cleanliness. For these reasons, increasing the number of private rooms in the medical wards was seen by patients as the ideal solution to their privacy and security problems.

Many non-Kuwaiti staff members stated that the working atmosphere in the medical department did not support incident reporting. They felt that neither the hospital leadership nor the laws and regulations supported non-Kuwaiti healthcare professionals when mistakes occurred. The reporting culture was seen as punitive by non-Kuwaitis, yet as overly permissive of mistakes by the Kuwaiti staff members and patients. Patients thought that there was a lack of responsibility and accountability in the department and that managers responded very permissively to staff mistakes. To encourage incident reporting and learning from errors, the staff generally suggested promoting/rewarding staff members participating in any quality improvement or patient safety initiatives rather than focusing on each error as a disciplinary issue.

Lack of proper feedback and follow-up by management after submitting an incident report was declared the main factor contributing to incidents not being reported. Staff were not motivated to report incidents as doing so was seen as not leading to significant departmental improvements. The staff believed that the incident reporting system should be reformed to support communication with departmental management and short- and long-term improvements to quality and safety. Some participants also emphasised the importance of simplifying the 
incident report form as they believed that completing the current form required excessive administrative efforts from them.

\section{Discussion}

This qualitative study provides insight into the PSC in one Kuwaiti medical department and, uniquely in the Arab/GCC countries context and incorporates multiple healthcare professional groups and patients' views. The data obtained suggests multiple constraints on PSC promotion in the department, specifically related to workload, communication, the healthcare environment and incident reporting. Kuwaiti and nonKuwaiti participants held different views about the department's response to errors and expatriate staff members' clinical skills. Participant suggestions as to how the diverse constraints of PSC implementation could be addressed included interventions at the individual (e.g. training and orientation) and organisational (e.g. increasing staff numbers, providing paid overtime and developing interdepartmental policies) levels.

The current staff members' perspectives were similar to those reported by a recent qualitative study conducted in two accredited public hospitals in Kuwait. ${ }^{5}$ For example, both studies suggested that patient safety was not prioritised in daily practice due to staff and resource shortages. However, unlike that study, the staff and patients in the current study suggested more training and orientation to improve staff awareness of patient safety. This difference in findings may be attributed to accountability differences between the two settings - the hospitals studied by $\mathrm{Al}$ Hamid et al. were accredited, while the hospital in the current study was not. ${ }^{5}$ Another difference of note involved the presence (or lack thereof) of supportive leadership. Whether by implementing an accreditation programme, offering supportive leadership or targeting other factors, it seems that healthcare organisations with a positive PSC have mechanisms that support decisive action. ${ }^{19}$ The perspectives of the current staff members were also more wide-spread. For example, studies conducted in hospitals in the USA have identified significant associations between low staff numbers (and associated increases in work demand) and adverse patient outcomes..$^{20}$ Maintaining optimal communication levels between healthcare staff members was found to be a key determinant of the PSC in hospital settings in a Chinese study. ${ }^{21}$ In addition, the healthcare environment's key role in promoting PSC has been emphasised in many studies. ${ }^{22,23}$ Other studies have suggested that feedback should be routine after incidents have been reported and should include information regarding response to the report in question. ${ }^{24,25}$

This study's findings also suggest that the surrounding healthcare environment and how staff behave and communicate with the patients played a crucial role in determining patient perceptions of departments' PSC. This finding is not confined to this study; previous studies conducted in other settings have revealed similar results. For example, a study conducted in oncology care settings found that patients valued the physical environment as an essential factor in having a safe healthcare experience. ${ }^{26}$ Another study investigating PSC in outpatient clinics in China reported that most participants linked poor organisational safety culture with the presence of a poor staff-patient relationship. ${ }^{27}$

A novel aspect of this study was the fact that it explored differences in how Kuwaiti and nonKuwaiti participants perceive responses to errors and expatriate staff's clinical skills. It seems that the damaged relationship and trust between citizens and expatriates in Kuwait caused by recently increasing public and political demands to control the number of expatriates in the workforce might influence the way PSC is perceived in the department by different national groups. ${ }^{28}$ This pattern has been seen elsewhere. For example, a study conducted in a university hospital in the conflict-ridden Eastern Democratic Republic of Congo also found that national politics affected healthcare. ${ }^{29}$ It is critical to examine differences between these groups given the large number of expatriate healthcare professionals in Kuwait. Evidence strongly suggests that an organisational safety culture is constituted by a mixture of safety subcultures and that the presence of contrasting views between these subcultures can compromise the achievement of organisation-wide safety goals. ${ }^{30,31}$ This evidence suggests that the differences between Kuwaiti and expatriate healthcare professionals should be explored more in-depth to identify how these differences might influence working practices. This information should be used to inform developing interventions to address cultural differences as part of PSC initiatives. ${ }^{32,33}$

This study showed that no single measure could work as a panacea for promoting PSC in this department. In this context, policymakers should think about interventions that target reform at different levels and include concerns about the healthcare system in general, specific staff behaviours and the healthcare environment. These interventions should be carried out in a coordinated manner to implement the Kuwaiti national patient safety standards against a backdrop of solidly-established PSC. Future research 
should focus on evaluating the approaches suggested by the study participants. This is no simple task and attention must be paid to both the outcomes and process of change.

This study has several strengths. Participants included a range of healthcare professionals and patients. While both groups' views were similar in many ways, patient participants focused more on issues related to the healthcare environment and staff behaviour. Another strength of this study is the iterative analysis of field notes and audio recordings throughout data collection, which allowed the researchers to adapt the semi-structured interview schedule for later interviews based on discussions in earlier interviews. The interviewer's status as both 'insider' and 'outsider' may have both helped and hindered the interviews. ${ }^{34}$ Since the interviewer previously worked in the hospital, he had good connections and relationships with the hospital leadership. These relationships facilitated obtaining ethical approval and access to the study's participants.

On the contrary, the interviewer's former employment as a hospital council member may have prompted participants to conceal some of the system's weaknesses. The interviewer's 'insider' status may have been an issue in data analysis regarding potential preconceived assumptions about the department's PSC status. Furthermore, the interviewer's Kuwaiti nationality may have negatively affected the openness of the interviews conducted with non-Kuwaiti participants. These hindrances were mitigated via the team's balance and reflective team discussions, which encouraged the constant considerations of alternative explanations in data interpretation. As a qualitative study, participant views cannot be considered generalisable, but they provide insight into perceptions and attitudes in the context under investigation. English was the second language for all participants, except for those who only spoke Arabic. This fact may have affected their ability to express their views about the department's PSC. Despite these limitations, faceto-face interviews enabled the participants to express their opinions about patient safety in their own words and gave them privacy to share their personal stories. ${ }^{17}$

\section{Conclusion}

To the best of the researchers' knowledge, this qualitative study is the first to consider both patients' and a wide range of healthcare professionals' perspectives of PSC in a GCC hospital setting. This study revealed multiple factors related to workload, communication, the healthcare environment and reporting incidents that hinder promoting a positive PSC in the studied departments. The presence of numerous constraints suggests that multiple interventions should be implemented that target both individual and organisational levels to promote PSC in the department. Further research is required to examine the effectiveness as well as the practicality of the suggested interventions.

\section{CONFLICT OF INTEREST}

The authors declare no conflicts of interest.

\section{FUNDING}

The first author received a scholarship from the government of Kuwait to do this work.

\section{References}

1. Agency for Healthcare Research and Quality. Hospital Survey on Patient Safety Culture: User's guide. Rockville, USA: US Department of Health and Human Services. 2016. 15(16)-0049-EF.

2. Elmontsri M, Almashrafi A, Banarsee R, Majeed A. Status of patient safety culture in Arab countries: A systematic review. BMJ Open 2017; 7:e013487. https://doi.org/10.1136/ bmjopen-2016-013487.

3. Najjar S, Baillien E, Vanhaecht K, Hamdan M, Euwema M, Vleugels A, et al. Similarities and differences in the associations between patient safety culture dimensions and self-reported outcomes in two different cultural settings: A national crosssectional study in Palestinian and Belgian hospitals. BMJ Open 2018; 8:e021504. https://doi.org/10.1136/bmjopen-2018-021504.

4. Alqattan H, Morrison Z, Cleland J. A narrative synthesis of qualitative studies conducted to assess patient safety culture in hospital settings. Sultan Qaboos Univ Med J 2019; 19:91-9. https://doi.org/10.18295/squmj.2019.19.02.002.

5. Al Hamid A, Malik A, Alyatama S. An exploration of patient safety culture in Kuwait hospitals: A qualitative study of healthcare professionals' perspectives. Int J Pharm Pract 2020; 28:617-25. https://doi.org/10.1111/ijpp.12574.

6. Nieva VF, Sorra J. Safety culture assessment: A tool for improving patient safety in healthcare organisations. Qual Saf Health Care 2003; 12:ii17-23. https://doi.org/10.1136/qhc.12.suppl_2.ii17.

7. Listyowardojo T, Ray-Sannerud B, Turk E, Lyons M, Pytte M, Leyshon S, et al. Mixed methods: Improving the assessment of safety culture in healthcare. From: www.dnvgl.us/publications/ mixed-methods-improving-assessment-of-safety-culture-inhealthcare-109113 Accessed: Jun 2020.

8. King E, Coldham T. Patients and carers can be our service design consultants. From: www.england.nhs.uk/blog/tina-coldham/ Accessed: Jun 2020

9. Davis RE, Sevdalis N, Neale G, Massey R, Vincent CA. Hospital patients' reports of medical errors and undesirable events in their health care. J Eval Clin Pract 2013; 19:875-81. https://doi. org/10.1111/j.1365-2753.2012.01867.x.

10. Alqattan H, Cleland J, Morrison Z. An evaluation of patient safety culture in a secondary care setting in Kuwait. J Taibah Univ Med Sci 2018; 13:272-80. https://doi.org/10.1016/j.jtum ed.2018.02.002

11. Ministry of Health, Kuwait. Accreditation standards for hospitals in Kuwait. Medical care version 5. From: cdhpage.com/wpcontent/uploads/2015/12/Check-The-Medical-Care-NationalAccreditation-Standards-for-Hospitals.pdf Accessed: Jun 2020. 
12. Malterud K, Siersma VD, Guassora AD. Sample size in qualitative interview studies: Guided by information power. Qual Health Res 2016; 26:1753-60. https://doi.org/10.1177/1049732315617444.

13. Josselson R. Interviewing for qualitative inquiry: A relational approach. New York, USA: Guilford Press, 2013.

14. Maxwell JA. Qualitative research design: An interactive approach, 3rd ed. Thousand Oaks, USA: Sage Publications, 2013.

15. Saunders B, Sim J, Kingstone T, Baker S, Waterfield J, Bartlam B, et al. Saturation in qualitative research: Exploring its conceptualization and operationalization. Qual Quant 2018; 52:1893-907. https://doi.org/10.1007/s11135-017-0574-8.

16. Graneheim UH, Lundman B. Qualitative content analysis in nursing research: Concepts, procedures and measures to achieve trustworthiness. Nurse Educ Today 2004; 24:105-12. https://doi.org/10.1016/j.nedt.2003.10.001.

17. Hays DG, Singh AA. Qualitative inquiry in clinical and educational settings. New York, USA: Guilford Press, 2011.

18. Korstjens I, Moser A. Series: Practical guidance to qualitative research. Part 4: Trustworthiness and publishing. Eur J Gen Pract 2018; 24:120-4. https://doi.org/10.1080/13814788.2017. 1375092.

19. Wachter RM, Pronovost P, Shekelle P. Strategies to improve patient safety: The evidence base matures. Ann Intern Med 2013; 158:350-2. https://doi.org/10.7326/0003-4819-158-5-201303050-00010.

20. Carayon P, Gurses AP. Nursing workload and patient safety-A human factors engineering perspective. In: Hughes RG, Ed. Patient safety and quality: An evidence-based handbook for nurses. Rockville, USA: Agency for Healthcare Research and Quality, 2008.

21. Zhu J, Li L, Li Y, Shi M, Lu H, Garnick DW, et al. What constitutes patient safety culture in Chinese hospitals? Int J Qual Health Care 2012; 24:250-7. https://doi.org/10.1093/ intqhe/mzs010.

22. Pelzang R, Johnstone M-J, Hutchinson AM. Culture matters: Indigenizing patient safety in Bhutan. Health Policy Plan 2017; 32:1042-8. https://doi.org/10.1093/heapol/czx042.

23. Wang Y, Liu W, Shi H, Liu C, Wang Y. Measuring patient safety culture in maternal and child health institutions in China: A qualitative study. BMJ Open 2017; 7:e015458. https://doi. org/10.1136/bmjopen-2016-015458
24. Antonacci AC, Lam S, Lavarias V, Homel P, Eavey RD. Benchmarking surgical incident reports using a database and a triage system to reduce adverse outcomes. Arch Surg 2008; 143:1192-7. https://doi.org/10.1001/archsurg.143.12.1192.

25. Francis R. Report of the Mid Staffordshire NHS Foundation Trust public inquiry: Executive summary. London, UK: The Stationery Office, 2013.

26. Browall M, Koinberg I, Falk H, Wijk H. Patients' experience of important factors in the healthcare environment in oncology care. Int J Qual Stud Health Well-being 2013; 8:20870. https:// doi.org/10.3402/qhw.v8i0.20870.

27. Liu C, Liu W, Wang Y, Zhang Z, Wang P. Patient safety culture in China: A case study in an outpatient setting in Beijing. BM] Qual Saf 2014; 23:556-64. https://doi.org/10.1136/bmjqs-20 13-002172.

28. Toumi H. Deep rifts emerge in Kuwait over expatriates' status. From: gulfnews.com/world/gulf/kuwait/deep-rifts-emerge-inkuwait-over-expatriates-status-1.1964054 Accessed: Jun 2020.

29. Labat F, Sharma A. Qualitative study exploring surgical team members' perception of patient safety in conflict-ridden Eastern Democratic Republic of Congo. BMJ Open 2016; 6:e009379. https://doi.org/10.1136/bmjopen-2015-009379.

30. Xuanyue M, Yanli N, Hao C, Pengli J, Mingming Z. Literature review regarding patient safety culture. J Evid Based Med 2013; 6:43-9. https://doi.org/10.1111/jebm.12020.

31. Sutcliffe KM, Paine L, Pronovost PJ. Re-examining high reliability: Actively organising for safety. BMJ Qual Saf 2017; 26:248-51. https://doi.org/10.1136/bmjqs-2015-004698.

32. Doval E, Doval O. Issues of standardisation concerning organisational culture in change management. 32nd Annual Congress of ARA Proceedings 2008; 978:374-7.

33. Mannion R, Davies H. Understanding organisational culture for healthcare quality improvement. BMJ 2018; 363:k4907. https:// doi.org/10.1136/bmj.k4907.

34. Tinker C, Armstrong N. From the outside looking in: How an awareness of difference can benefit the qualitative research process. Qual Rep 2008; 13:53-60. 